\title{
Innovative Biotechnological Approaches for the Upgradation of Diverse Scientific Disciplines
}

\author{
Kennady Vijayalakshmy ${ }^{1 *}$, Meenakshi Virmani ${ }^{2}$, Vikas Choudhiry $^{3}$, Rajesh Dalal ${ }^{4}$, \\ Upendra Lambe ${ }^{5}$, Ranjeet Verma ${ }^{6}$ and J. Manimegalai ${ }^{5}$
}

${ }^{1}$ Department of Veterinary Physiology, ${ }^{2}$ Animal Physiology Division, ${ }^{4}$ Department of Animal Nutrition, ${ }^{5}$ Department of Animal Biotechnology, Lala Lajpat Rai University of Veterinary \& Animal Sciences, Hisar, 125004, Haryana, India

${ }^{3}$ Department of Veterinary Gynaecology and Obstetrics, NDUAT, Faizabad-224229, India

${ }^{6}$ Department of Animal Reproduction, Indian Veterinary Research Institute, Bareilly, Uttar Pradesh, India

*Corresponding author

\section{Keywords}

Agriculture; Biotechnology; Classification: Environment; Pharmaceutics; Veterinary

Article Info

Accepted:

15 May 2019

Available Online:

10 June 2019

\section{Introduction}

\section{Branches and application of biotechnology}

Biotechnology is a multidisciplinary network involving chemists, biologists, engineers and many other specialists. Its scope is enormous. It is playing a major role in serving the mankind.
Biotechnology is a major branch of science that plays a critical role in various different fields. The major fields where biotechnology plays a progressive role include agriculture, veterinary sciences, chemical technology, food technology, environmental studies and pharmaceutical industry. There are various other branches or fields where biotechnological principles are efficiently applied. Biotechnology is classified into four major groups depending on the field for which it has been used. The present review depicts the application of biotechnology in various scientific fields. 
fixation in plants and vaccine production from plants.

The main aim is to increase the food production through below mentioned ways.

Agro-chemical based agriculture

Organic agriculture

Genetically engineered crop-based agriculture

Advantages of genetic modification in crops:

Made crops more tolerant to abiotic stresses

Reduce reliance on chemical pesticides

Helped to reduce poor harvest losses

Increased efficiency of mineral usage by plants

Enhanced nutritional value of food e.g. Vitamin A rich rice (CAST, 1999).

In addition to these genetic modifications has been used to create tailor-made plants to supply alternative resources to industries, in the form of starches, fuels and pharmaceuticals. Production of pest resistant plants reduces the usage of pesticide.

A toxin called $\mathrm{Bt}$ toxin, where the gene has been cloned from the bacteria Bacillus thuringiensis. This $\mathrm{Bt}$ toxin gene has been cloned and expressed in plants to provide resistance to insects without the need for insecticides, in effect created a bio-pesticide. Bio-fungicides have been used in both the phylloplane and rhizosphere to suppress fungal infection in plants.

Species of Bacillus and Pseudomonas have been successfully used as seed dressings to control certain soil borne plant diseases (Wieczorek, 2003).

\section{Mechanism of action of Bacillus thuringenisis}

Some strains of $B$. thuringenesis produce protein that kills larval stage insects such as Lepidopterans (tobacco budworm, armyworm), Coleopterans (beetles) and Dipterans (flies and mosquitoes). B.thuringenesis forms protein crystals (Wieczorek, 2003). These crystals contain a toxic insecticidal protein. Eg.Bt cotton, bt corn, tomato, rice, potato and soyabean. Another important contribution is the conservation of germplasm through storage in liquid nitrogen $-196^{\circ} \mathrm{C}$ (cryopreservation) (CAST, 1999).

\section{Veterinary sciences}

This sector broadly covers development of improved breed of animals with higher milk production, higher animal productivity and with other desirable characteristics, animal feed, embryo transfer, developing vaccines etc. The leading modern biotechnology applications in veterinary sciences are:

Biotechnology derived veterinary vaccines A number of different vaccines produced using biotechnology are already in use and many more are at different stages of development. Their benefits include decreasing the risk of reversion to virulence of live vaccines and of interference with antibodies induced by passive immunization, avoiding contamination with other viruses, reducing damage during storage and other aspects. The great advantage of these vaccines is that they can be used in systems for differentiating infected from vaccinated animals (Differentiate Infected from Vaccinated Animals - DIVA).

As DIVA vaccines make it possible to differentiate vaccinated animals from animals exposed to virus strains, they allow diseases 
to be eradicated from a country much faster and more economically than by using conventional eradication methods.

One of the first diseases to be successfully eradicated using the DIVA strategy was Aujeszky's disease (Carlos and Boronto, 2008).

Immunocastration - Biotechnology-derived veterinary vaccines are being used not only to control infectious diseases, but also to increase productivity by modulating hormones or immune system functions, as well as for immunocastration, ectoparasite control, etc (Wray and Woodward, 1990).

Growth promoters or immune boosters - In recent years, biomolecules have been developed that have proven effective in inducing endogenous genes that promote growth and boost the immune system (innate and acquired immunity).

Faster the growth and development of young animals, it also increases rates of survival, weight gain and synchronization of the animal population (Carlos and Boronto, 2008). Metabolic modifiers have been tested with particular success in aquatic organisms (fish and shrimp) and been found to significantly improve production and to reduce the use of antibiotics and chemicals.

Veterinary diagnostic systems - numerous diagnostic techniques have been used to detect various diseases such as Immunoenymatic techniques, rapid diagnostic strip tests, various types of PCR, microarray techniques and proteomics (Wray and Woodward, 1990).

Prebiotics and probiotics - The leading prebiotics are non-digestible oligosaccharides includes fructo-oligosaccharides (FOS), galacto-oligosaccharides (GOS), malto- oligosaccharides (MOS) and xylooligosaccharides (XOS). The most typical prebiotics are FOS and inulin and they are sold widely for use in humans and monogastric animals. Symbiotics are a combination of probiotics and prebiotics that have a beneficial effect on the host's health by improving the survival and implantation of probiotics in the gastrointestinal tract by selectively stimulating their activity and/or growth (Wray and Woodward, 1990). Scientific literature documents that the consumption of probiotics, prebiotics or symbiotic improves the intestinal flora, prevents and rehabilitates diarrheal diseases, improves the composition of blood lipids, reduces cholesterol, improves blood pressure, increases calcium absorption and retention, modulates immune functions and reduces the risk of colon cancer.

In case of animal biotechnology, biotechnological principles are used in In-vivo fertilization, embryo transfer, Hormoneinduced superovulation and embryo splitting in farm animals that helps in rapid multiplication of animals with superior genotypes. Production of transgenic animals for increased milk, growth rate, resistance to diseases etc. (Carlos and Boronto, 2008).

\section{Pharmaceutical Industry}

The recombinant DNA technological processes have made immense impact in the area of healthcare by enabling mass production of safe and more effective therapeutic drugs (Walsh, 2007).

Using certain biotechnological techniques can produce various types of enzymes and hormones, where production of Insulin hormone from E. coli plasmid with the help of rDNA technology. Recombinant vaccines can also be produced, e.g. Hepatitis B vaccine and monoclonal antibodies. Other important 
pharmaceutical products produced by immunosuppressant's and anti-tumor microorganisms are cholesterol lowering compounds (Gaisser and Nusser, 2007). agents or statins, enzyme inhibitors,

Branches and application of biotechnology
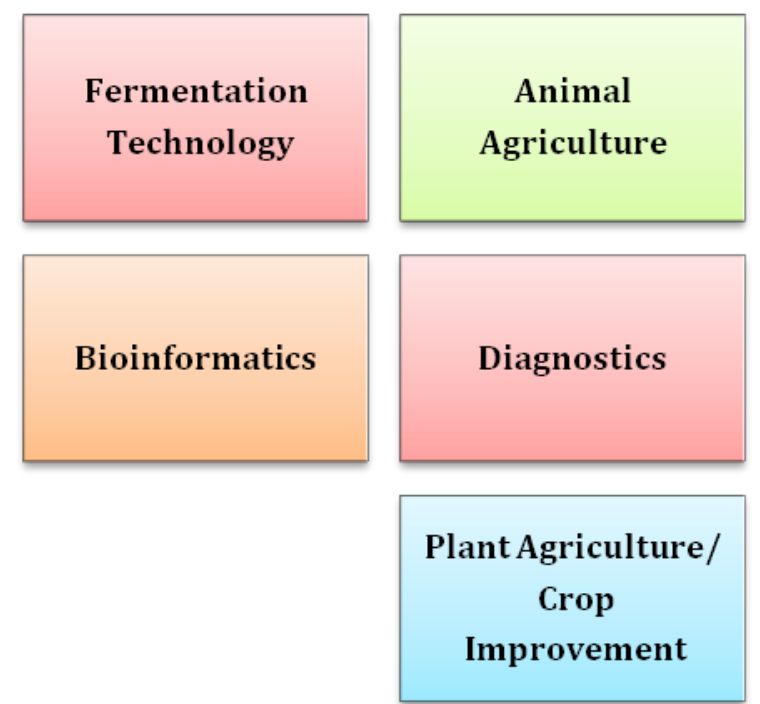
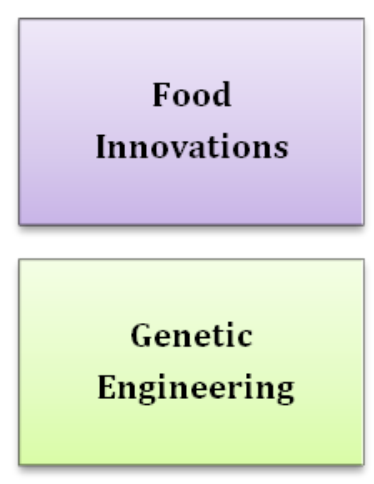

Genomics/

Proteomics
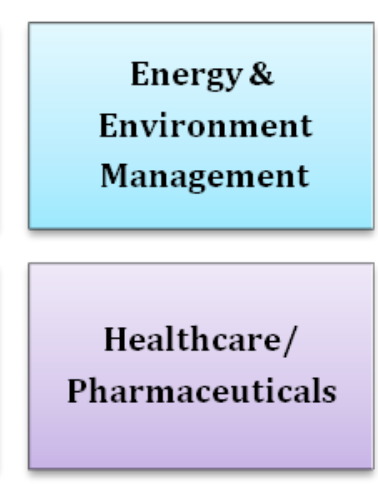

Classification

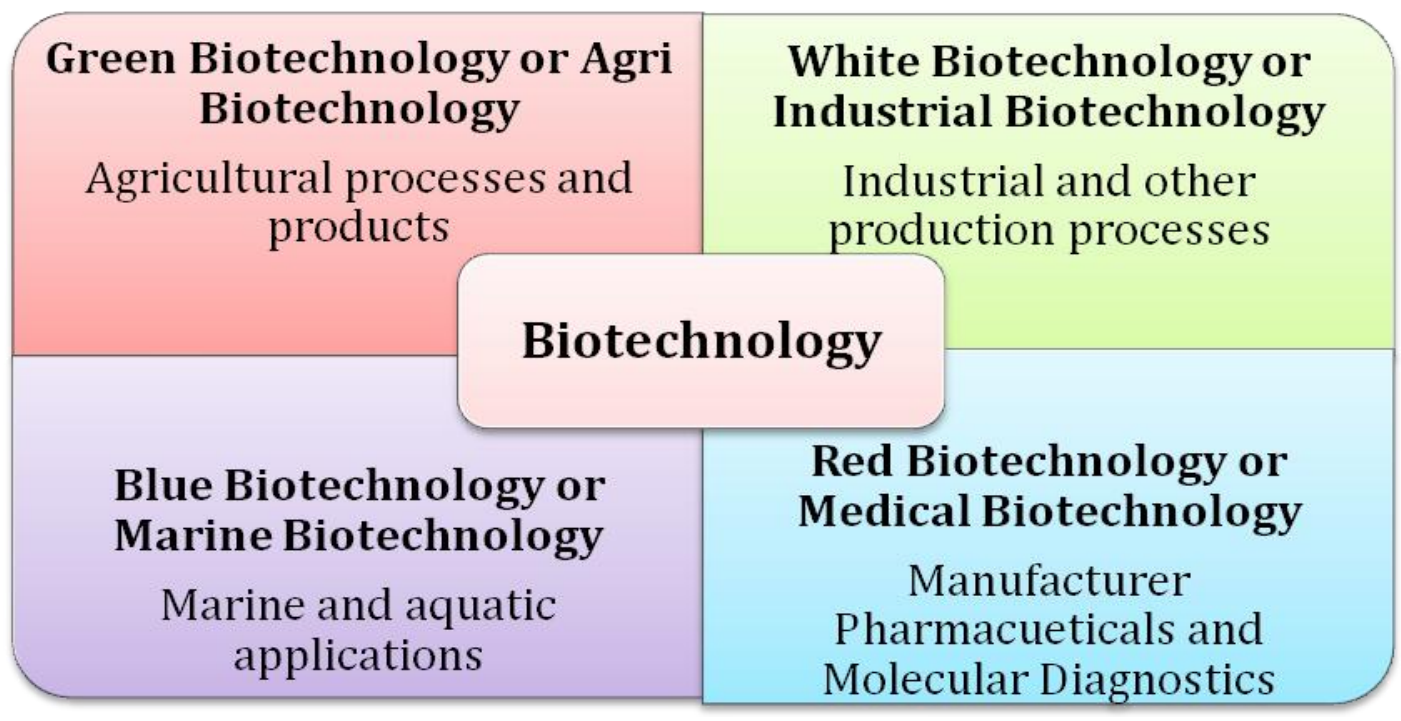

\section{Food industry}

Biotechnology in the food-processing sector makes use of microorganisms for the preservation of food. And for the production of a range of value-added products such as enzymes, flavor compounds, vitamins, microbial cultures and food ingredients (Gaisser and Nusser, 2007).

Biotechnology applications in the foodprocessing sector, therefore, target the selection and manipulation of microorganisms with the objective of improving process 
control, product quality, safety, consistency and yield, while increasing process efficiency. Hybridization is also used for the improvement of yeasts involved in baking, brewing and in beverage production.

For e.g. Saccharomyces cerevisiae strains have been researched for improved fermentation, processing and bio preservation abilities, and for capacities to increase the wholesomeness and sensory quality of wine (Food Insight, 1997).

Biotechnology can be used for monitoring food safety, prevention and diagnosis of food borne illness. Detection of mycotoxins, aflatoxins, fumonisins, ocharotoxins, zearalonone and deoxynivalenol etc. - DNA based identification code system on relevant polymorphisms at nucleotide level (Walsh, 2007).

The fermentation bioprocess is the major biotechnological application in food processing. Fermentation bioprocessing makes use of microbial inoculants for enhancing properties such as the taste, aroma, shelf life, safety, texture and nutritional value of foods. Usage of starter culture for spontaneous inoculation to accelerate fermentation processes e.g. Engineered baker`s yeast

\section{Chemical industry}

A sustainable alternative for renewable sources can be produced in chemical industry. Greatly reduce dependence on nonrenewable fuels and other sources. Production of compounds such as ethanol, lactic acid, glycerin, citric acid, gluconic acid, acetone from micro-organisms mainly from bacteria (Gaisser and Nusser, 2007). Production of enzymes can be done from bacteria, fungi, yeasts and algae for human food and animal feed (Walsh, 2007). Production of fuel can be done from cheap, less useful and abundant substrates from sugarcane, bagasse and wood ${ }^{[2]}$. Mineral extraction can be done from lowgrade ores. A great adventure of biotechnology associated with chemical industry includes the Biocatalyst, which is very much helpful in waste degrading process.

Microalgae are used to some extent in bio treatment of wastewaters, as aquaculture feeds, bio fertilizers and soil inoculants (Food Insight, 1997). Potentially, they can be used for removing excess carbon dioxide from the environment. Extremophilic enzymes, or extremozymes, are finding increasing industrial use because of their ability to withstand extremes of temperatures and other conditions.

\section{Environmental studies}

Using microorganisms, efficient sewage treatment can be done. Degradation of petroleum and management of oil spills can also be managed. Detoxification of wastes and industrial effluents can also be done (Walsh, 2007). Bio-control of plant diseases, insects and pests provide ecofriendly approach that can prevent pollution. Environment friendly detergents that reduce eutrophication in the environment can also be prepared.

In conclusion, biotechnology has got a tremendous group of advantages associated with almost all the branches of science. In recent days, the contributions of biotechnology in various fields are highly tremendous and the future development and achievements by biotechnology will be highly appreciable. Biotechnology can make meticulous changes in all branches of science that will be highly helpful in empowering the developmental strategies. 


\section{References}

Carlos, G., and Borroto. 2008. Biotechnology and its Application to Veterinary Science. Conf. OIE, 231-240.

Council for Agricultural Science and Technology (CAST). 1999. "Applications of Biotechnology to Crops: Benefits and Risks", Issue Paper, Number 12, Dec.

Food Insight. 1999. Myths and Facts about Food Biotechnology. Food Insight, September/October, PP 2-3.

Gaisser, N., and Nusser, M.2007. The role of biotechnology in pharmaceutical drug design. Z Evid Fortbild Qual
Gesundhwes; 104(10):732-7.

Walsh, G. 2007. Pharmaceutical Biotechnology - Concepts and Applications. Wiley, Chichester, West Sussex, UK; p. 48.

Wieczorek, A. (2003). Use of biotechnology in agriculture - benefits and risks. Honolulu (HI): University of Hawaii. 6 p.

Wray, C., and Woodward, M. J. (1990). Biotechnology and veterinary science: production of veterinary vaccines. Revue scientifique at technique (International Office of Epizootics) 9 (3), 779-794.

\section{How to cite this article:}

Kennady Vijayalakshmy, Meenakshi Virmani, Vikas Choudhiry, Rajesh Dalal, Upendra Lambe, Ranjeet Verma and Manimegalai J. 2019. Innovative Biotechnological Approaches for the Upgradation of Diverse Scientific Disciplines. Int.J.Curr.Microbiol.App.Sci. 8(06): 21512156. doi: https://doi.org/10.20546/ijcmas.2019.806.255 